\title{
Influence of Age-Related Changes in Nitric Oxide Synthase-Expressing Neurons in the Rat Supraoptic Nucleus on Inhibition of Salivary Secretion
}

\author{
By
}

\author{
Takehiko TANAKA, Yoshitaka TAMADA and Fumihiko SUWA
}

\author{
Department of Anatomy, Osaka Dental University, 8-1 Kuzuhahanazono-cho, Hirakata-shi, Osaka 573-1121, Japan
}

\begin{abstract}
Age-related inhibition of salivary secretion has been demonstrated in rats, and the nitric oxide (NO) present in the supraoptic nucleus (SON) and the medial septal area has been reported to play an inhibitory role in the regulation of salivary secretion. In the present study, we investigated the age-related changes occurring in the NO synthase (NOS)-expressing neurons in the SON, which is related to the production of NO, and discussed the interrelation between the age-related changes in the NOS-expressing neurons and the age-related inhibition of salivary secretion. Nissl staining and reduced nicotinamide adenine dinucleotide phosphate diaphorase (NADPH-d) histochemistry were performed for young adult and aged rats. Quantitative analysis was also performed using the Nissl-stained and NADPH-dpositive neurons. Although the numbers of the Nissl-stained neurons did not change, significant age-related increases were detected in cell number, cell size and reactive density of the NADPH-d-positive neurons. Therefore, the production of NO in the SON neurons increased with age.

We concluded that the age-related increase in the NO in the SON might be a factor that contributes to the agerelated inhibition of salivary secretion.
\end{abstract}

- Received for Publication, December, 20, $2007-$

Key Words: Nitric oxide synthase, NADPH-diaphorase, Supraoptic nucleus, Salivary secretion, Aged rat

\section{Introduction}

Age-related inhibition of salivary secretion has been demonstrated in rats ${ }^{11}$. Recently, many lines of evidences have indicated that nitric oxide (NO) is involved in the regulation of salivary secretion $^{2,3)}$. In the supraoptic nucleus (SON) and the medial septal area of rats, NO synthase (NOS)expressing neurons were identified, and NO synthesis by these neurons was demonstrated ${ }^{4,5}$. Additionally, a few papers have suggested that the presence of NO in the SON and the medial septal area may play an inhibitory role in the regulation of salivary secretion ${ }^{6,7)}$. However, no studies are available regarding the interrelation between the age-related changes in the NOS-expressing neurons in the SON and the inhibition of salivary secretion.

In the present study, we investigated the agerelated changes in the NOS-expressing neurons, which are related to the production of $\mathrm{NO}$, and discussed the interrelation between the age-related changes in the NOS-expressing neurons and the age-related inhibition of salivary secretion.

\section{Materials and Methods}

For this study, 12 male Sprague-Dawley (SD) rats were used; 6 young adult male SD rats (2 months old, young adult group, average body weight $220 \mathrm{~g}$ ) and 6 aged male SD rats (24 months old, aged group, average body weight $920 \mathrm{~g}$ ).

These animals were housed in a temperaturecontrolled room $\left(23^{\circ} \mathrm{C}\right)$ and maintained in individual cages under constant light/dark cycles (lights were switched on at 0800 and switched off at 2000) with free access to food and water. The present study was conducted according to the Guidelines for Animal Research at Osaka Dental University (ODU) and the National Institute of Health (NIH) Guidelines for the Care and Use of Laboratory Animals. Further, this study was also approved by

Correspondence to: Fumihiko Suwa, D.D.S, Ph.D., Department of Anatomy, Osaka Dental University, 8-1 Kuzuhahanazono-cho, Hirakata-shi, Osaka 573-1121, Japan. E-mail: suwa@cc.osaka-dent.ac.jp 


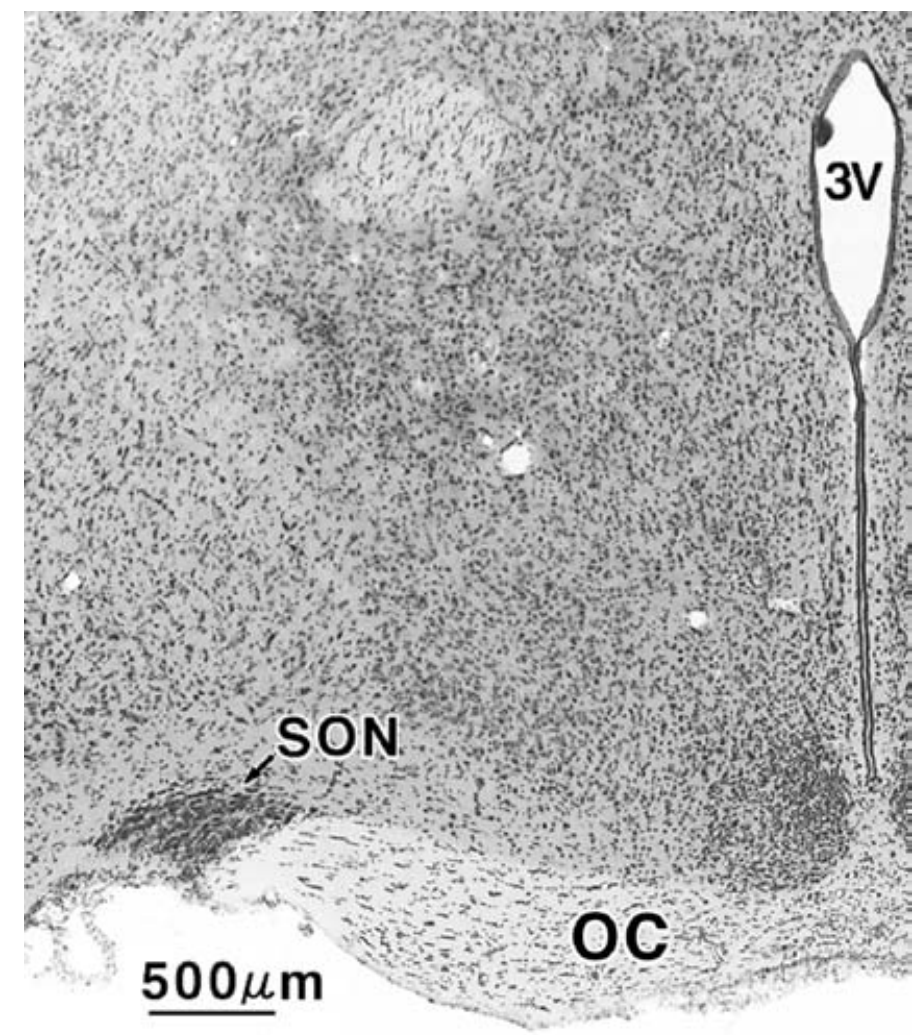

Fig. 1. Nissl-stained sections in the hypothalamic region of the young adult group.

The SON that is located in the hypothalamic region of the brain is a small paired nucleus just above the OC. SON, supraoptic nucleus; $3 \mathrm{~V}$, third ventricle; OC, optic chiasm.

the Animal Research Committee for ODU (approval number 07-02013).

All of the animals were sacrificed by injecting an overdose of pentobarbital $(50 \mathrm{mg} / \mathrm{kg}$, i.p.; Nembutal $^{\circledR}$, Dainippon Pharmaceutical Co. Ltd). They were immediately perfused via the left cardiac ventricle with $0.1 \mathrm{M}$ phosphate-buffered saline (PBS) and fixed in a fixative containing $4 \%$ paraformaldehyde, which was adjusted to $\mathrm{pH} 7.4$ with $0.1 \mathrm{M}$ phosphate buffer (PB). After the removal of the brain, tissue blocks, including hypothalamic tissue, were postfixed in the same fixative for $24 \mathrm{~h}$ at $4^{\circ} \mathrm{C}$, and subsequently immersed in $20 \%$ sucrose in $0.1 \mathrm{M} \mathrm{PB}$ for $24 \mathrm{~h}$ at $4^{\circ} \mathrm{C}$.

Serial, free-floating frontal sections $(20-\mu \mathrm{m}$ thick $)$ were prepared using a cryostat (Microm HM500$\mathrm{OM}^{\circledR}$, Zeiss) based on the locations of the anterior commissure, the optic chiasm and the optic tract. These sections were stored in PBS containing $0.1 \%$ Triton X-100. All histochemical reactions were processed using the free-floating sections, and all specimens were marked in order to differentiate between individual animals. From each group, 3 animals were employed for the staining procedures that were performed as follows (refer to sections 1 and 2). For all staining procedures, all the specimens were processed simultaneously in the same solution for the same duration.

After the staining procedures, all the sections that were subjected to Nissl staining and NADPH-d histochemistry were observed under a light microscope (Microphot-FXA ${ }^{\circledR}$, Nikon). The sections were photographed, and all data were converted into the digital format. Additionally, quantitative and statistical analyses were performed according to the methods described in sections 3 and 4.

\section{Nissl staining}

To investigate the age-related changes in the SON neurons, serial sections obtained from 3 animals from each group were used for Nissl staining in a $0.1 \%$ cresyl violet (Cresyl violet ${ }^{\circledR}$, Chroma) solution.

\section{NADPH-d histochemistry}

To investigate the age-related changes in the NOS-expressing neurons in the SON, the serial 


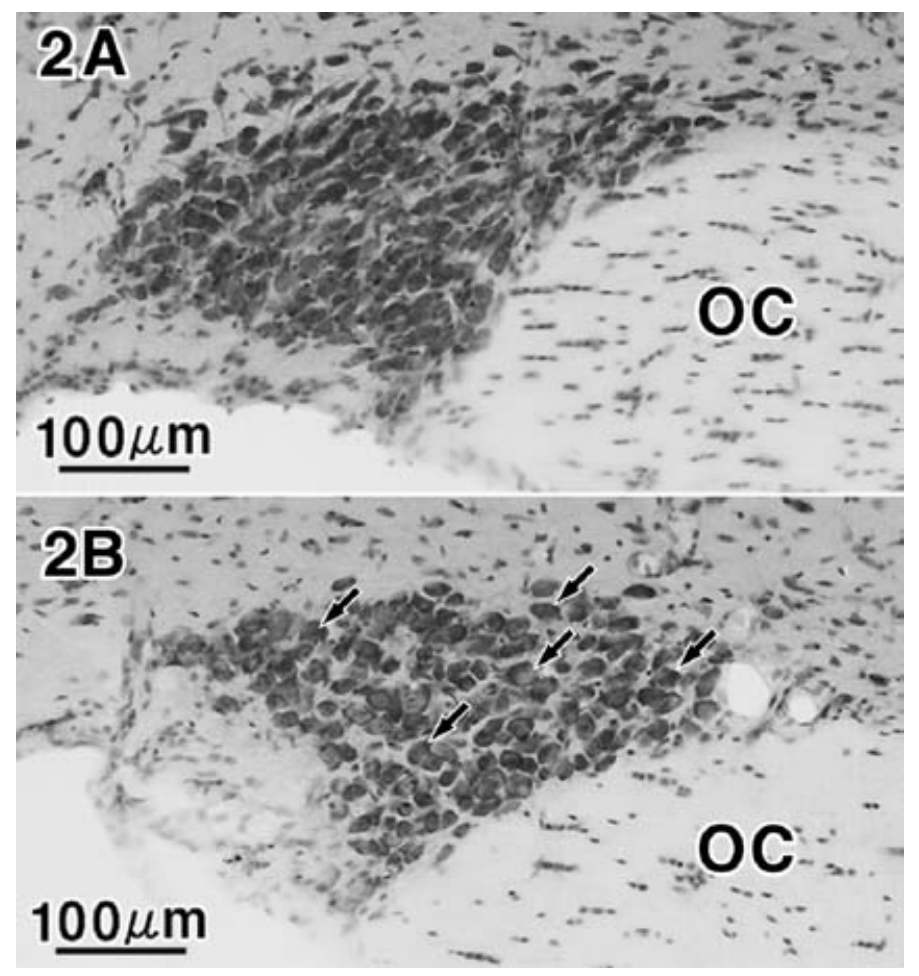

Fig. 2. Nissl-stained sections of the SON (Higher magnification).

In the higher-magnification view, many Nissl-stained neurons were observed in the SON. In comparison with the SON of the young adult group (2A), many large neurons (arrows) were observed in the SON of the aged group (2B). OC, optic chiasm.

sections obtained from 3 animals of each group were used for NADPH-d histochemistry. For the detection of NADPH-d activity, the sections were incubated in a reaction buffer $(5 \mathrm{mg}$ of $\beta$-NADPH (Merck) and $1 \mathrm{mg}$ of nitroblue tetrazolium (Merck) in $10 \mathrm{ml}$ of $0.1 \mathrm{M}$ PBS containing $0.1 \%$ Triton $\mathrm{X}-100$ ) for $1 \mathrm{~h}$ at $37^{\circ} \mathrm{C}$. The reaction in PBS was then arrested.

\section{Quantitative analysis}

Using a computer-assisted image processing system, the cell number and cell size of the Nisslstained and the NADPH-d-positive neurons were examined. Further, the reaction density of the NADPH-d-positive neurons in the bilateral SON of 3 animals from each group was measured. Five specimens of the middle portion of the SON were randomly selected from individual animals. For the measurement of cell number, the number of Nissl-stained and NADPH-d-positive neurons was counted under a light microscope. For measuring cell size and reaction density, 10 neurons were randomly selected from individual specimens and measurements were obtained using the Scion Image beta 4 version software (Scion Corporation). For measuring the reactive density of the NADPH-dpositive neurons, the reactive density of the NADPH-d-positive cytoplasm was measured in terms of optical density (OD) after conversion of the RGB digital data into grayscale data.

\section{Statisitical procedure}

The value for each animal was represented as mean \pm standard deviation. Student's $t$ test was used to test statistical significance $(n=6$, $\mathrm{p}<0.001)$.

\section{Results}

\section{Nissl staining}

A number of Nissl-stained neurons were observed in the SON of the young adult and aged groups (Fig. 2A, 2B).

Employing a computer-assisted image processing system, no significant differences were observed in the number of Nissl-stained neurons between the 2 groups (young adult group, $127.7 \pm 2.1$ : aged group, $133.0 \pm 7.4)$. However, on comparison of both the groups (Fig. 3A, 3B), a significant increase was ob- 

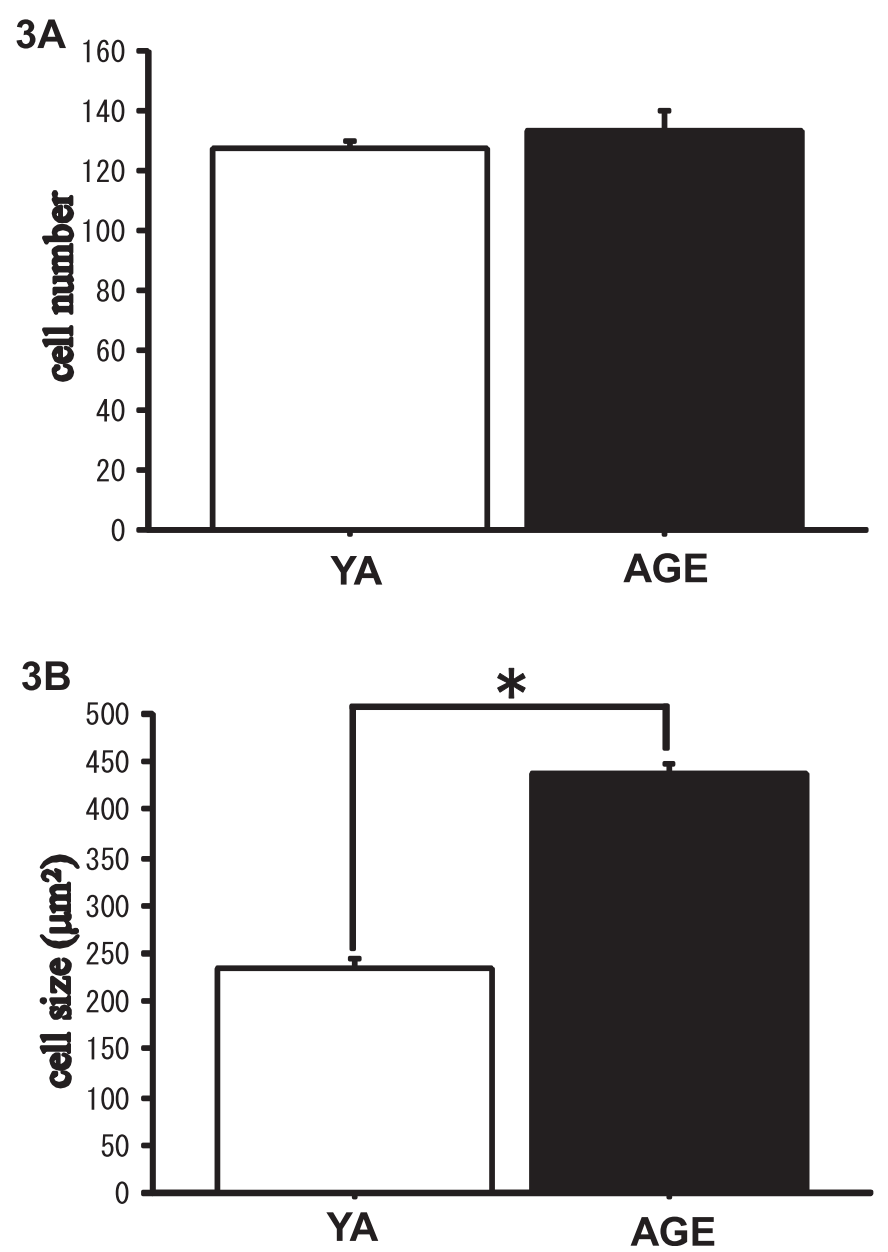

Fig. 3. Quantitative analysis of the Nissl-stained neurons.

No significant differences were detected in cell number (3A). However, age-related significant differences were detected in the size of the Nissl-stained neurons $(\mathrm{n}=6, * \mathrm{p}<0.001)(3 \mathrm{~B})$. YA, young adult group; AGE, aged group.

served in the size of the Nissl-stained neurons (young adult group, $1224.5 \pm 12.4 \mu \mathrm{m}^{2}$ : aged group, $\left.455.3 \pm 25.3 \mu \mathrm{m}^{2}\right)$.

\section{NADPH-d histochemistry}

In the young adult and aged groups, NADPH-d activity was observed in the magnocellular neurons of the SON (Fig. 4A, 4B). In the young adult group, NADPH-d-positive neurons were found mainly in the dorsal part of the SON: very few neurons with weak NADPH-d activity were observed in the ventral part of the SON (Fig. 4A). Further, in the aged group, many magnocellular neurons with strong NADPH-d activity were observed throughout the entire SON (Fig. 4B).

Comparison of the groups by using a computerassisted image processing system, revealed a significant increase in the cell number (young adult group, $51.7 \pm 2.7$ : aged group, $80.2 \pm 4.4$ ), cell size (young adult group, $234.5 \pm 13.0 \mu \mathrm{m}^{2}$ : aged group, $460.7 \pm 38.1 \mu \mathrm{m}^{2}$ ) and reactive density (young adult group, $120.0 \pm 5.5$ OD: aged group, $190.7 \pm$ 9.5 OD) of NADPH-d-positive neurons in the aged group (Fig. 5A, 5B, 5C).

\section{Discussion}

In this section, we first discussed the experimental procedures and then the interrelation between the age-related changes in the NOS-expressing neurons and the age-related inhibition of salivary secretion.

\section{Experimental procedures}

NO was first identified as an endotheliumderived relaxing factor ${ }^{8)}$. Recently, it has been demonstrated that it may play the role of a neuro- 

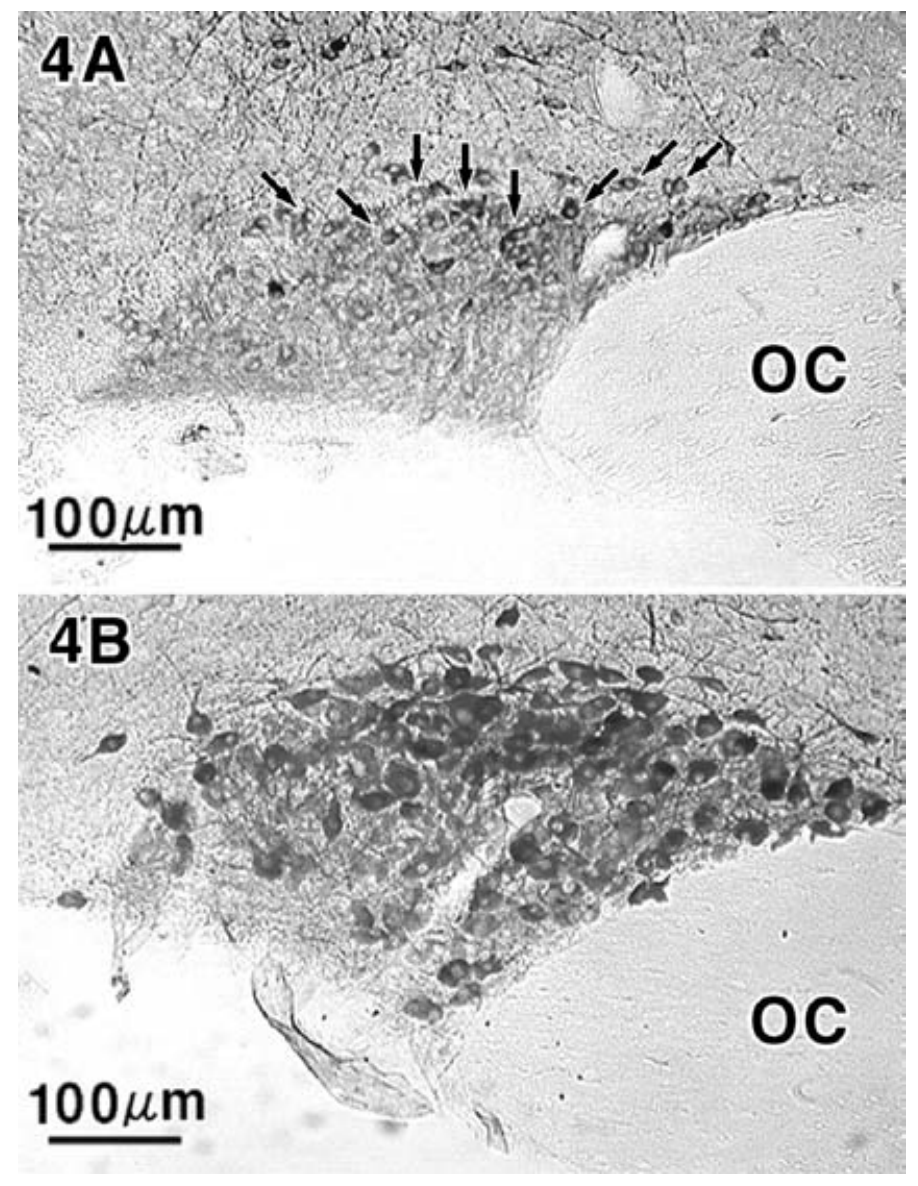

Fig. 4. NADPH-d histochemistry in the SON.

In the young adult group, NADPH-d-positive neurons (arrows) were mainly located in the dorsal part of the SON (4A). While in the aged group, many magnocellular neurons with strong NADPH-d activity were observed throughout the entire SON (4B). OC, optic chiasm.

nal messenger, i.e., that of a second messenger and neurotransmitter in the central and peripheral nervous systems ${ }^{9,10)}$. Endogenously, NO is formed by NOS, which transforms arginine into NO and citrulline $^{9)}$, and NADPH-d has been demonstrated as a reliable histochemical maker for $\operatorname{NOS}^{11,12)}$. Additionally, it was clarified that all NOS-expressing neurons in the SON exhibit NADPH-d activity and that all NADPH-d-positive cell bodies in the SON are NOS-positive ${ }^{13)}$. Therefore, in the present study, we employed the NADPH-d histochemistry and not NOS immunohistochemistry for identifying the NOS-expressing neurons in the SON.

In order to obtain exact data for quantitative analysis, all the specimens were marked in order to distinguish between the individual animals from which they were obtained. Further, all the specimens were processed simultaneously in the in the same solution for the same duration.
2. Interrelation between the age-related changes in the NOS-expressing neurons and the age-related inhibition of salivary secretion

It has been reported that the injecting an NO synthase inhibitor $\left(\mathrm{N}^{\omega}\right.$-nitro-L-arginine-methyl ester; L-NAME) into the SON and the medial septal area cause an increase in salivary secretion, while injecting an NO donor (FK409) decreased salivary secretion $^{6,7)}$. Further, these reports also suggested that the NO in the SON and the medial septal area play inhibitory roles in the regulation of salivary secretion $^{6,7)}$.

Quantitative analysis of the cell number in the SON revealed no significant differences in the Nissl-stained neurons between the 2 groups: therefore, we concluded that there were no age-related changes in cell number in the SON. However, quantitative analysis of the NADPH-d-positive neurons, revealed significant age-related increases 

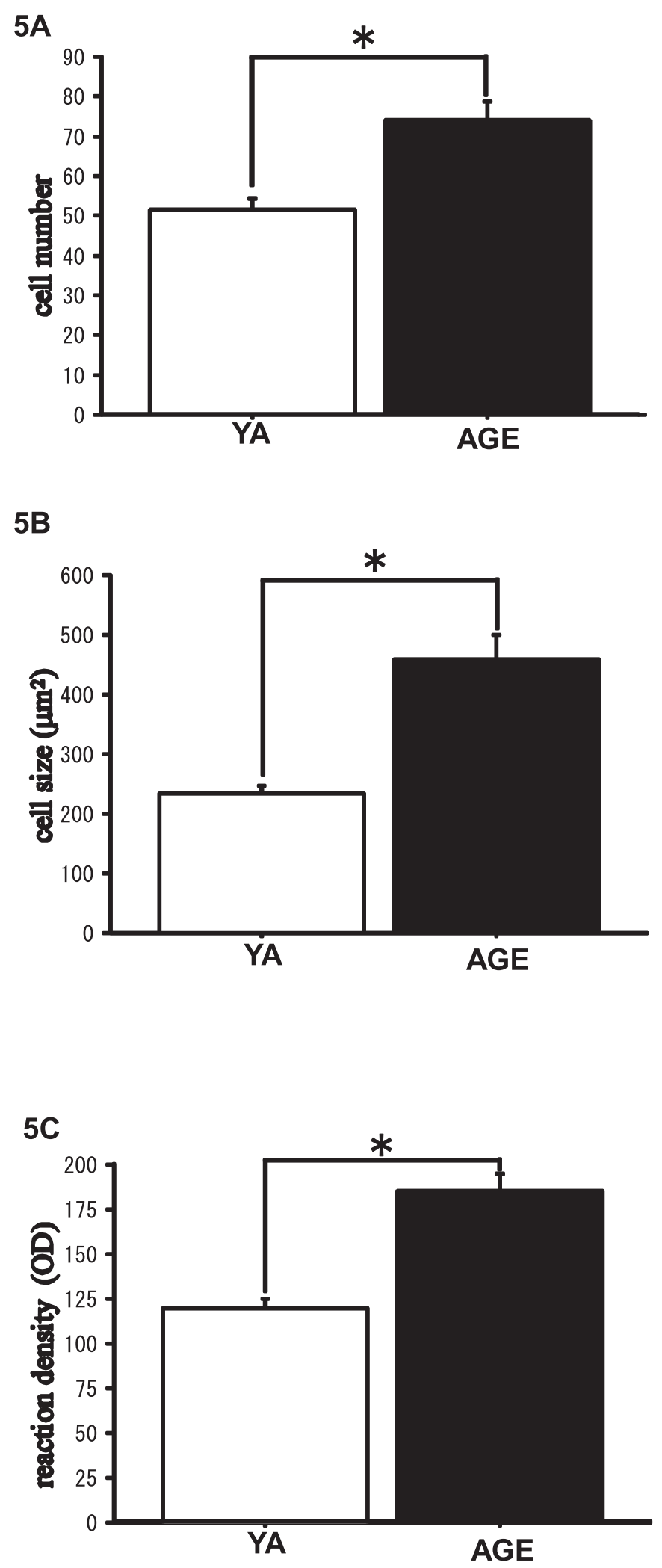

Fig. 5. Quantitative analysis of the NADPH-d-positive neurons.

Age-related significant differences were detected in cell number (5A), cell size (5B) and reaction density (5C) of NADPH-dpositive neurons $\left(\mathrm{n}=6,{ }^{*} \mathrm{p}<0.001\right)$. YA, young adult group; AGE, aged group. 
in cell number, cell size and reaction activity of the NADPH-d-positive neurons. Therefore, the NO in the SON was concluded to increase with age.

\section{Conclusion}

We concluded that the age-related increase in the NO in the SON might be a factor that contributes to the age-related inhibition of salivary secretion.

\section{Acknowledgments}

This study was performed using the Morphological Research Facilities, the Photograph Processing Facilities and the Laboratory Animal Facilities of the Institute of Dental Research, Osaka Dental University. The authors wish to express their sincere gratitude to all staff members of the Department of Anatomy for their invaluable assistance. This study was presented at the 512th Annual Meeting of the Osaka Odontological Society, February 16, 2008, Hirakata, Japan.

\section{References}

1) Bodner L, Gorsky M: Parotid gland secretion of the aging rat. Arch Gerontol Geriatr 1996; 22:63-69.

2) Rettori V, Lomniczi A, Elverdin JC, Suburo A, Faletti A, Franchi A, McCann SM: Control of salivary secretion by nitric oxide and its role in neuroimmunomodulation. Ann N Y Acad Sci 2000; 917:258-267.

3) Lomniczi A, Suburo AM, Elverdin JC, Mastronardi CA, Diaz S, Rettori V, McCann SM: Role of nitric oxide in salivary secretion. Neuroimmunomodulation 1998; 5:226-233.

4) Sugaya K, McKinney M: Nitric oxide synthase gene expression in cholinergic neurons in the rat brain examined by combined immunocytochemistry and in situ hybridization histochemistry. Brain Res Mol Brain Res 1994; 23:111-125.

5) Miyagawa A, Okamura H, Ibata Y: Coexistence of oxytocin and NADPH-diaphorase in magnocellular neurons of the paraventricular and the supraoptic nuclei of the rat hypothalamus. Neurosci Lett 1994; 171:13-16.

6) Saad WA, Guarda IF, Camargo LA, dos Santos TA, Simoes S, Guarda RS: Novel evidence that nitric oxide of the medial septal area influences the salivary secretion induced by pilocarpine. Life Sci 2002; 70:2403-2412.

7) Saad WA, Gutierrez LI, Guarda IF, Camargo LA, dos Santos TA, Simoes S, Guarda RS: Nitric oxide of the supraoptic nucleus influences the salivary secretion, sodium renal excretion, urinary volume and arterial blood pressure induced by pilocarpine. Life Sci 2004; 74:1593-1603.

8) Furchgott RF, Zawadzki JV: The obligatory role of endothelial cells in the relaxation of arterial smooth muscle by acetylcholine. Nature 1980; 288:373-376.

9) Snyder SH: Nitric oxide: first in a new class of neurotransmitters. Science 1992; 257:494-496.

10) Moncada S, Palmer RM, Higgs EA: Nitric oxide: physiology, pathophysiology, and pharmacology. Pharmacol Rev 1991; 43:109-142.

11) Hope BT, Michael GJ, Knigge KM, Vincent SR: Neuronal NADPH diaphorase is a nitric oxide synthase. Proc Natl Acad Sci U S A 1991; 88:2811-2814.

12) Bredt DS, Snyder SH: Nitric oxide, a novel neuronal messenger. Neuron 1992; 8:3-11.

13) Okamura H, Miyagawa A, Takagi H, Esumi H, Yanaihara $\mathrm{N}$, Ibata Y: Co-existence of PACAP and nitric oxide synthase in the rat hypothalamus. Neuroreport 1994; 5:11771180. 
